\title{
WAYS OF UNEVEN ROAD SURFACE MODELLING USED IN THE VEHICLE DYNAMICS ANALYSIS
}

\author{
Szymon Tengler, Andrzej Harlecki \\ University of Bielsko-Biala \\ Department of Mechanics \\ Willowa Street 2, 43-309 Bielsko-Biala, Poland \\ tel.: +48338279405 \\ e-mail:stengler@ath.bielsko.pl,aharlecki@ath.bielsko.pl
}

\begin{abstract}
An important issue, which should be taken into account while analyzing the dynamics of vehicles moving along an uneven road surface, is the proper representation of its real shape. Two mathematical models of the road surfacecontinuous and discrete were considered in the method presented in this paper. These models were used in the computer simulations of dynamics of a special vehicle with a high gravity centre, which moved along an uneven road surface. The simulations were carried out using the worked out program package, in which four main modules could be differentiated such as: preprocessor, solver, postprocessor and $3 D$ animation. This package was implemented combined with the Blender graphics environment - in authors' opinion it is an innovative approach. Owing to this models of uneven road surface (theoretically of any shapes) can be built and they are input data for both the solver and animation modules. Additionally, the Blender tool can be used to prepare $3 D$ objects, which are used in the animation process of the moving model of a vehicle. According to the authors', developed program package developed on the basis of the mathematical models for the analyzed vehicle and road surface can be interesting for engineers designing special vehicles. By performing different types of the computer simulations, the engineers can improve the vehicle being built and reduce cost of its road test.
\end{abstract}

Keywords: vehicle dynamics, uneven road surface modelling, computer simulation

\section{Introduction}

When the vehicle dynamics is analysed, an essential issue while developing its mathematical model is the knowledge on forces and moments of the road surface, which act on the wheels. Mathematical models of tires of different complexity can be used to determine those forces and reaction moments. In the doctoral dissertation [5], which was basis for this article, three models of tires - Fiala, Dugoff-Ufelmann and Pacejka, have been used. In each of these models, it is assumed that the forces and moment of the road surface reactions are applied in one point - in the so-called contact point $C$. In the quoted doctoral dissertation, four algorithms - VectorCross, Plane, 4Points and Simple, were developed to determine a position of this point. Each of the algorithms mentioned is based on the known mathematical model representing the uneven road surface. Two such models, namely continuous and discrete ones, are presented in this article.

\section{The continuous model of the road surface}

In the continuous model, the Bicubic interpolation [3] was used for the mathematical representation of the road surface, thus for determining the three-dimensional interpolation surface. The input data are here the points in the three dimensional space - so called control points (interpolation nodes). The points located between these nodes are searched.

Let $P_{i, j}^{\prime}$ and $P^{\prime}$ mean the $x y$ plane projections of the control point $P_{i, j}\left(x_{i}, y_{i}, z_{i}\right)$ and the searched point $P\left(x_{P}, y_{P}, z_{P}\right)$ respectively, being on the interpolated surface, of which first two coordinates $x_{P}$ and $y_{P}$ are known. Then, the third coordinate $z_{P}$ of the searched $P$ point is determined by 
transforming the given grid cell specified by points $P_{i, j}^{\prime}, P_{i+1, j}^{\prime}, P_{i+1, j+1}^{\prime} P_{i, j+1}^{\prime}$, into the square of the side length equal to 1 (Fig. 1).

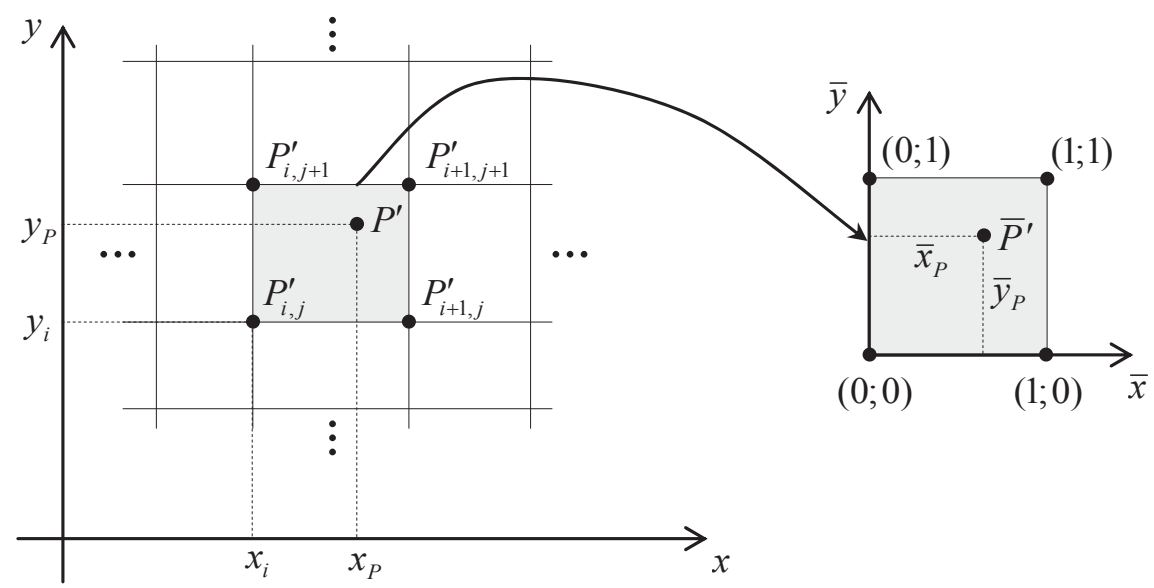

Fig. 1. Transforming the selected grid cell

After such transformations, coordinate $z_{P}$ is calculated according to the formula:

$$
z_{P}=f\left(x_{P}, y_{P}\right)=\bar{z}_{P}=\bar{f}\left(\bar{x}_{P}, \bar{y}_{P}\right)=\sum_{i=0}^{3} \sum_{j=0}^{3} a_{i, j} \bar{x}_{P}^{i} \bar{y}_{P}^{i},
$$

where:

$$
\left.\begin{array}{c}
\bar{x}_{P}=\frac{x_{P}-x_{i}}{x_{i+1}-x_{i}} \\
\bar{y}_{P}=\frac{y_{P}-y_{j}}{y_{i+1}-y_{j}}
\end{array}\right\} \in\langle 0 ; 1\rangle
$$

are the new coordinates of point $P$.

Formula (1) contains 16 unknown coefficients $a_{i, j}$, which are determined according to the method described in the doctoral dissertation [5].

From analyses made within the scope of the cited doctoral dissertation, it results that smooth interpolation surfaces are obtained when the continuous model is used, and they have an advantageous influence on efficiency of the calculation process performed in the scope of the analysis of the vehicle dynamics. However, the continuous model does not allow imitating unevenness of the road surface, which fragments are flat in some places (e.g. a vehicle drives through a speed bump on one side, and on the other it drives over the flat surface). In such a case, the discrete model should be taken into account.

\section{The discrete model of the road surface}

In the discrete model of the road surface developed by the author of the cited doctoral dissertation, it was assumed that this surface is modelled by the surface area built out of triangles (Fig. 2).

A number of triangles and their sizes are selected to represent the real shape of the road surface as accurately as possible.

The surface area made of the triangles can be defined on the basis of two sets:

1. $S^{(P)}=\left\{P_{1}\left(x_{1}, y_{1}, z_{1}\right), \ldots, P_{i}\left(x_{i}, y_{i}, z_{i}\right), \ldots, P_{n}\left(x_{n}, y_{n}, z_{n}\right)\right\}-$ a set of all points from which the triangles are built, where $P_{i}\left(x_{i}, y_{i}, z_{i}\right)$;

2. $S^{(v)}=\left\{\mathbf{v}^{(1)}, \ldots, \mathbf{v}^{(i)}, \ldots, \mathbf{v}^{(m)}\right\}$ - a set of 3-element vectors defining all the triangles, that is $\mathbf{v}^{(i)}=\left[\begin{array}{lll}v_{1}^{(i)} & v_{2}^{(i)} & v_{3}^{(i)}\end{array}\right]^{T}$, where $v_{1}^{(i)}, v_{2}^{(i)}, v_{3}^{(i)} \in\{1, \ldots, n\}$ are indexes of the points (from the $S^{(P)}$ set) 


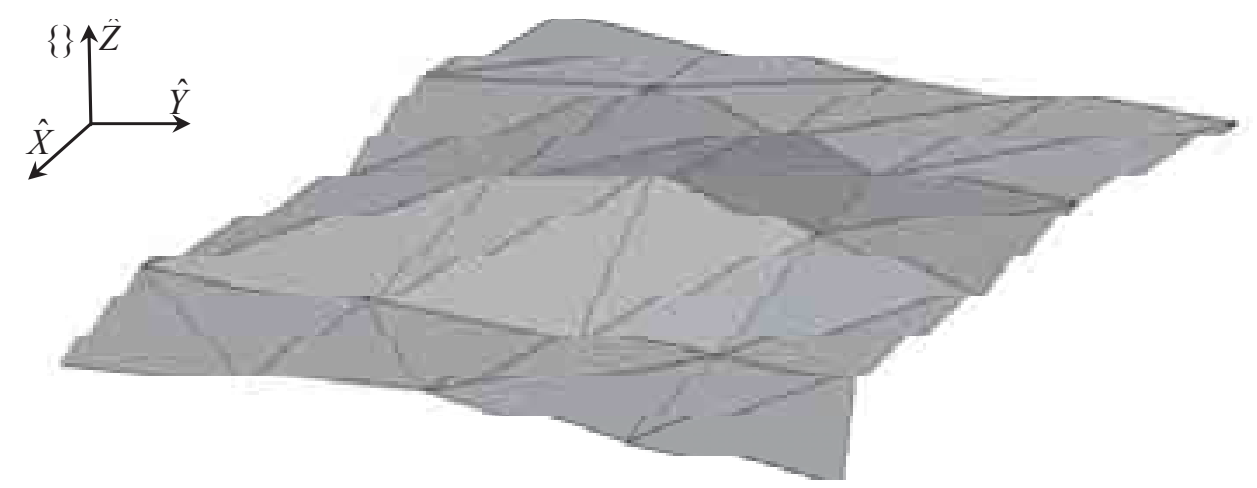

Fig. 2. Example of the road surface fragment modelled by triangles

being vertexes $i$-th of this triangle. It means mathematically that for each element of the $S^{(v)}$ set a mapping function, which indicates that the $\mathbf{v}^{(i)}$ element from the $S^{(v)}$ set describes a triangle of vertexes $P_{v_{1}^{(i)}}, P_{v_{2}^{(i)}}, P_{v_{3}^{(i)}}$, was determined in a form of: $\mathbf{v}^{(i)} \mapsto\left\{P_{v^{(i)}}, P_{v_{2}^{(i)}}, P_{v_{3}^{(i)}}\right\}$.

It should be emphasised that one element (a point) from the $S^{(P)}$ set can be a common element (a point) for two or more triangles simultaneously. As in the continuous model, a position of the $P\left(x_{P}, y_{P}, z_{P}\right)$ point being in the surface area in question is searched and its coordinates $x_{P}$ and $y_{P}$ are known (Fig. 3). The $z_{P}$ coordinate of point $P$ can be determined in a way described further in this article.

Let point $P$ be in the area of the $k$ triangle of vertexes $P_{v_{1}^{(k)}}, P_{v_{2}^{(k)}}, P_{v_{3}^{(k)}}$ (Fig. 3).

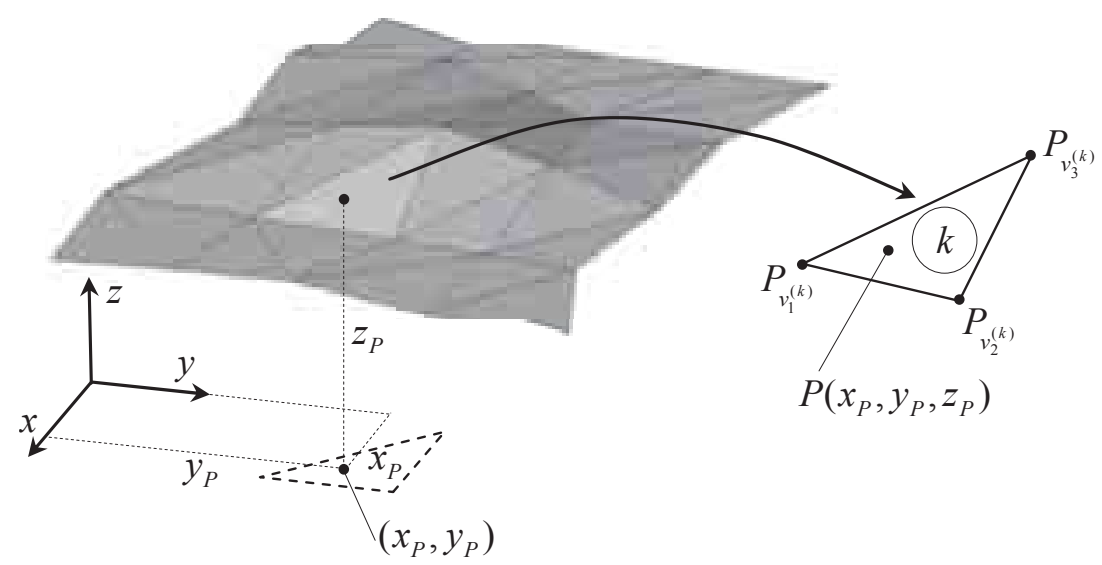

Fig. 3. Position of the searched point $P$ on the surface area modelled by the triangles

The vertexes of this triangle determine the plane of which the normal equation has the following form [1]:

$$
e_{x}^{(k)} x+e_{x}^{(k)} y+e_{x}^{(k)} z+\delta^{(k)}=0
$$

where:

$e_{x}^{(k)}, e_{y}^{(k)}, e_{z}^{(k)}$ - elements of the $\hat{\mathbf{e}}^{(k)}$ versor normal to the $k$ triangle surface area, $\delta^{(k)}=-\left(e_{x}^{(k)} x_{l}+e_{y}^{(k)} y_{l}+e_{z}^{(k)} z_{l}\right)$, where $l$ should be taken as any number from set $\left\{v_{1}^{(k)}, v_{2}^{(k)}, v_{3}^{(k)}\right\}$.

Versor $\hat{\mathbf{e}}^{(k)}$ can be determined by the vector product:

$$
\hat{\mathbf{e}}^{(k)}=\frac{\mathbf{p}_{1,2}^{(k)} \times \mathbf{p}_{1,3}^{(k)}}{\left|\mathbf{p}_{1,2}^{(k)} \times \mathbf{p}_{1,3}^{(k)}\right|},
$$

where:

$\mathbf{p}_{1,2}^{(k)}$ - the vector with the beginning in point $P_{v_{1}^{(k)}}$ and the end in point $P_{v_{2}^{(k)}}$,

$\mathbf{p}_{1,3}^{(k)}$ - the vector with the beginning in point $P_{v_{1}^{(k)}}$ and the end in point $P_{v_{3}^{(k)}}^{v^{(k)}}$. 
Having the plane equation determined in (2), the searched $z_{P}$ coordinate of the point $P$ can be determined from the formula:

$$
z_{P}=-\frac{e_{x}^{(k)} x_{P}+e_{y}^{(k)} y_{P}+\delta^{(k)}}{e_{z}^{(k)}},
$$

for $e_{z}^{(k)} \neq 0$, excluding the situation when the $k$ triangle plane is perpendicular to the $x y$ plane those cases do not concern this work.

In the described procedure, it was assumed that the vertexes of the triangle, on which there is point $P$, are known. However, identification of this triangle is not a trivial task. It becomes especially difficult in a case of computer simulations where short time of calculations is usually significant. Therefore, it is essential to develop an appropriate algorithm of the triangle identification of the surface area in question. The trivial solution of the triangle identification problem consists of searching the whole set of triangles $S^{(v)}$ and checking if the searched element is in the surface area of this triangle. In this case, for each triangle $(k=1, \ldots, m)$ the plane equation (2) should be determined, and it should be checked if the $P$ point is in its fragment specified by vertexes $P_{v_{1}^{(k)}}, P_{v_{2}^{(k)}}, P_{v_{3}^{(k)}}$. Such an algorithm does not belong to efficient regarding calculating, and because of three dimensionality, it may prove to be problematic. Much better results can be obtained by reducing the problem to a two dimensional issue and narrowing appropriately the set of the searched triangles. In this work, the developed algorithm was divided into two stages.

Stage I - reducing the problem to a two dimensional issue

Let $S^{(P)^{\prime}}=\left\{P_{1}^{\prime}, \ldots, P_{i}^{\prime}, \ldots, P_{n}^{\prime}\right\}$ mean a set of the points being projections of the points from the $S^{(P)}$ set on the $x y$ plane. A two dimensional (flat) map in which there are projections of the triangles of the area in question, is obtained in such a way. Then, a triangle is searched in this map; the triangle that is appropriate three points from the $S^{(P)}$ set, to which point $P^{\prime}$ is a projection of the $P$ point on the $x y$ plane (Fig. 4). When the vertexes of this triangle are known, there are also known vertexes determining a position of a triangle corresponding to it in the three dimensional space.

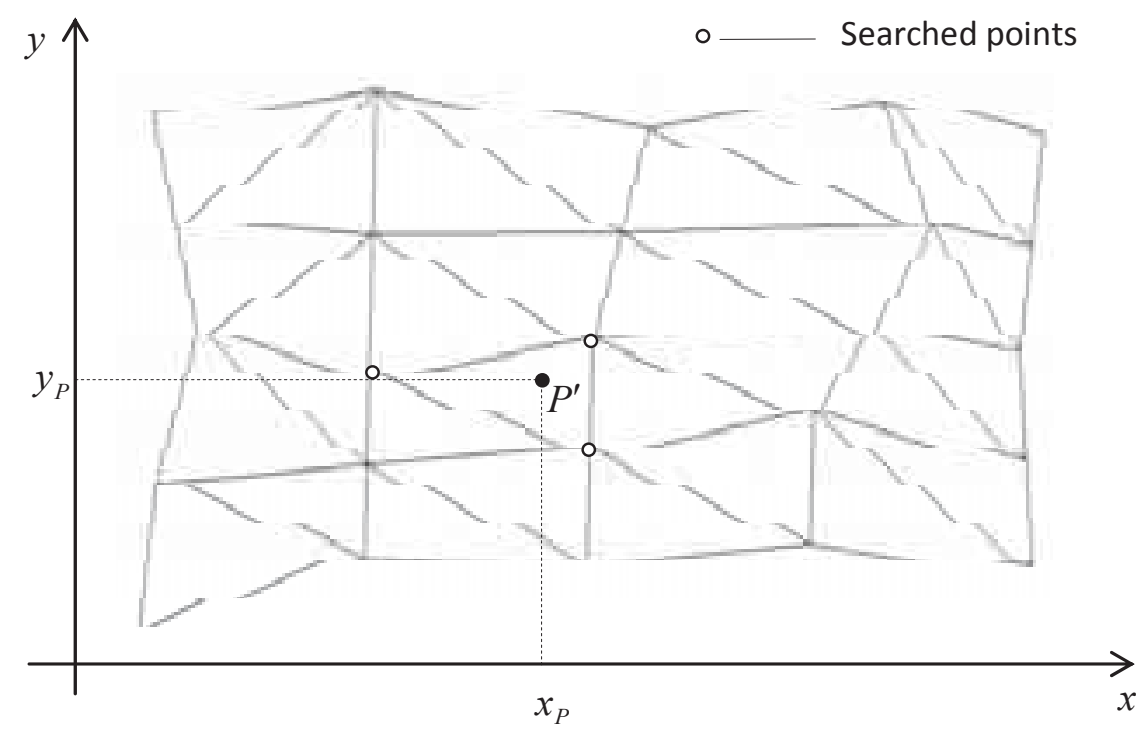

Fig. 4. Projection of the surface area on the xy plane

Stage II - limiting the search set

A distance of a $P^{\prime}\left(x_{i}, y_{i}\right)$ point from the $P^{\prime}\left(x_{P}, y_{P}\right)$ point is determined by the formula:

$$
d_{i}=\left|P^{\prime}-P_{i}^{\prime}\right|=\sqrt{\left(x_{P}-x_{i}\right)^{2}-\left(y_{P}-y_{i}\right)^{2}} .
$$

Let $P_{k}^{\prime}$ mean such a point of the $S^{(P)^{\prime}}$ set, of which the distance (5) is the shortest. This point 
will be called the nearest neighbour. Then, the hypothetical triangles, to which point $P^{\prime}$ may belong, are only those for which point $P_{k}^{\prime}$ is their common vertex (Fig. 5). There is a problem to find this point - a way of its searching can have a significant influence on efficiency of the algorithm in question. A typical solution based on determining the distance (5) for all $n$ points is not the optimal solution.

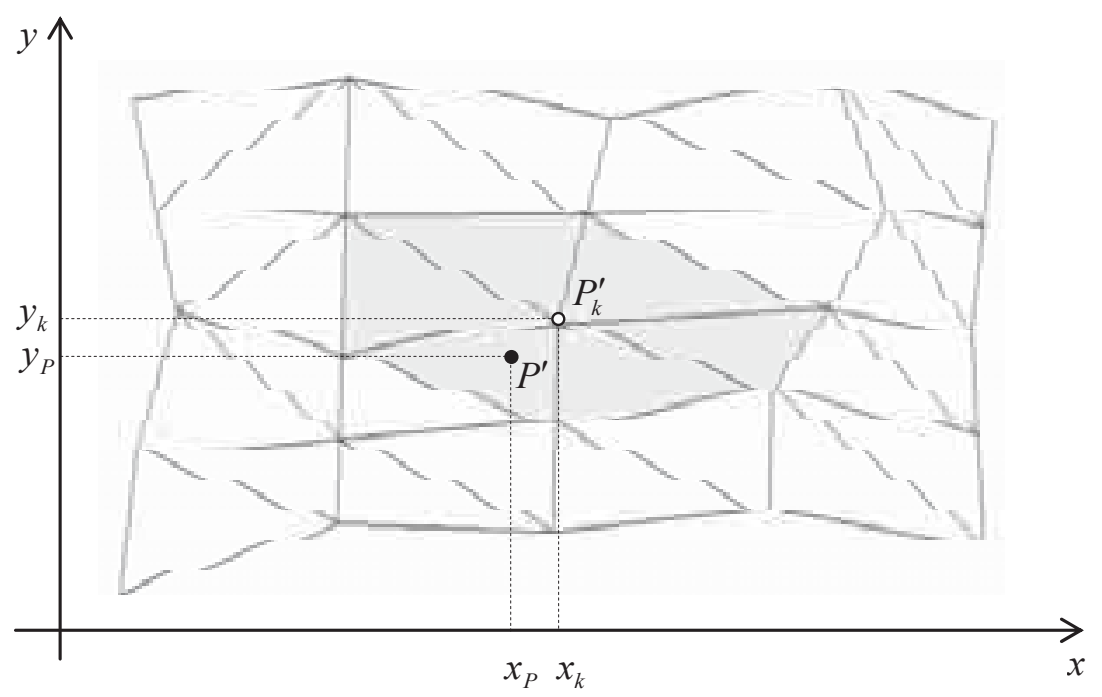

Fig. 5. Searched triangles (marked in grey)

In this work, an effective algorithm based on the data structure of the kd-tree type was used for searching the nearest neighbour (Nearest Neighbour Search, abbreviated to NNS). This algorithm was presented in details in the doctoral dissertation [5] on basis of the information contained in [2, 4]. The final identification process of the "appropriate" triangle of the surface area in question consists of specific steps, which are presented, in the diagram (Fig. 6).

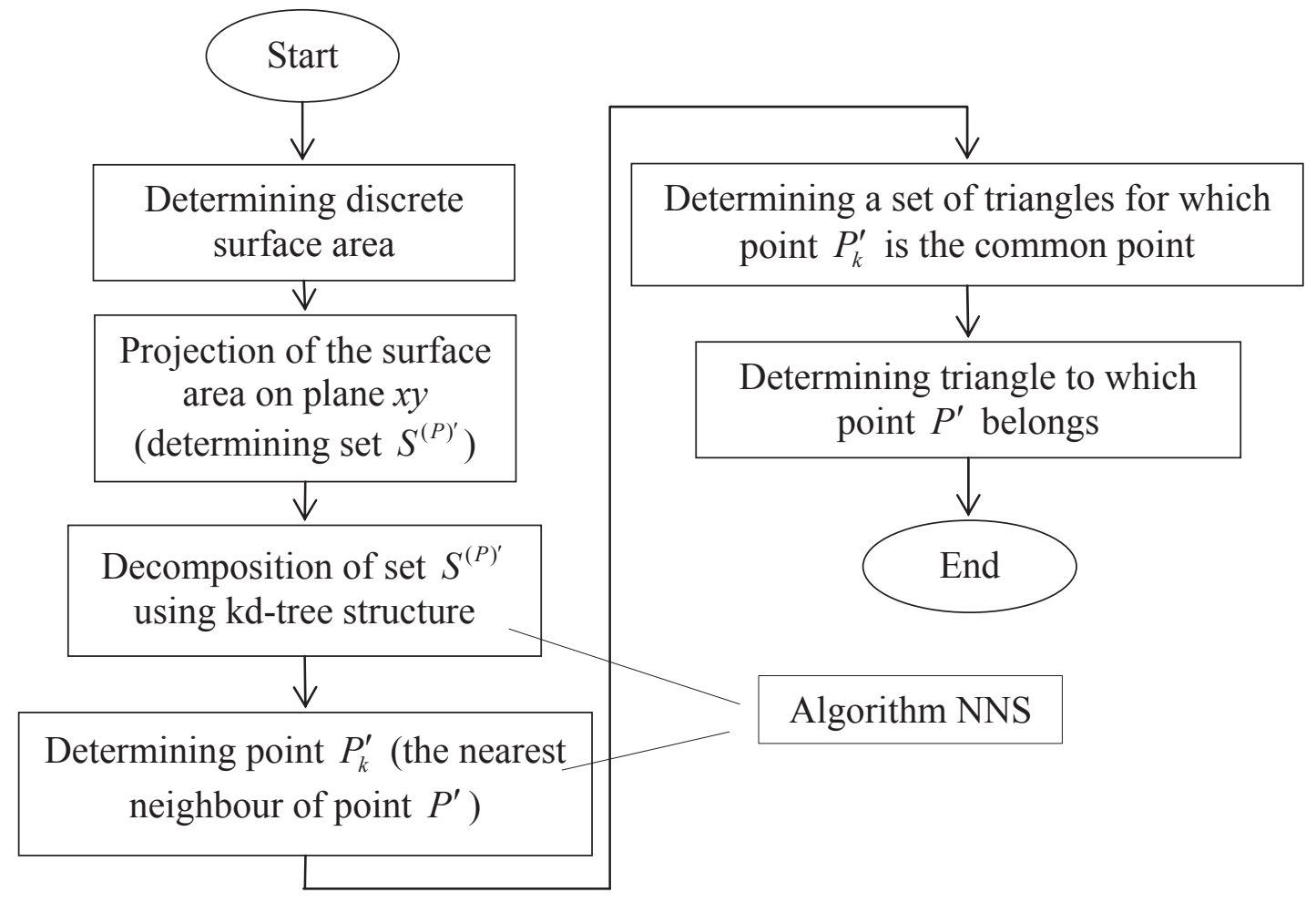

Fig. 6. Stages of triangle identification 
The presented procedure in the case of the surface area made of triangles can be used also for a surface area made of quadrangles (e.g. rectangles). An example of the surface area made of rectangles, which constitutes a model of the road surface, is presented in Fig. 7.

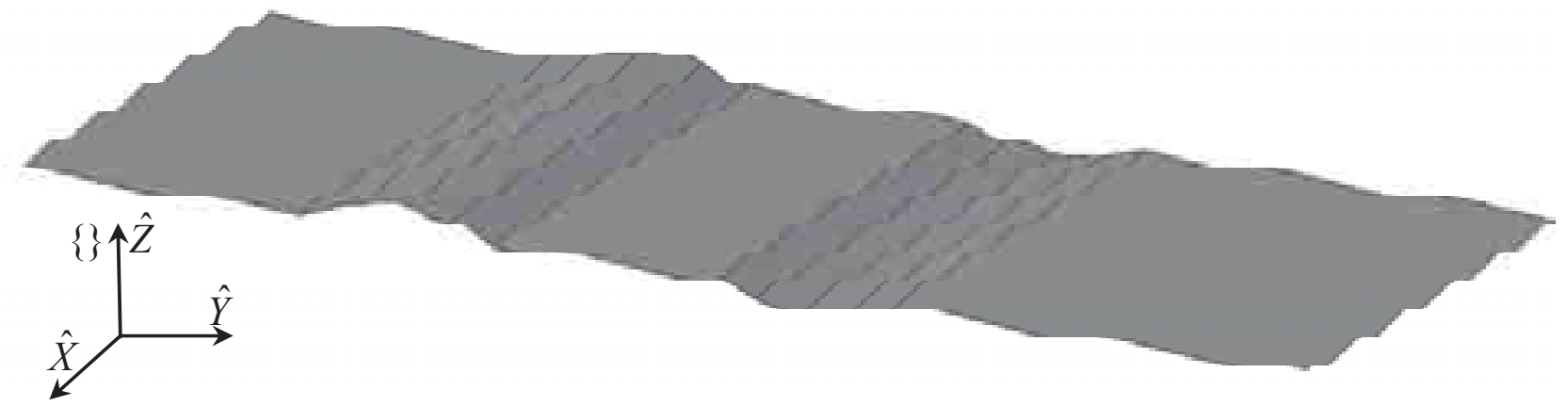

Fig. 7. Exemplary model of the surface made of rectangles

The surface areas made in such a way cannot contain „false quadrangles” (figures of which vertexes are not in the same plane). An exemplary model of the road surface containing "the false quadrangle" is presented in Fig. 8.

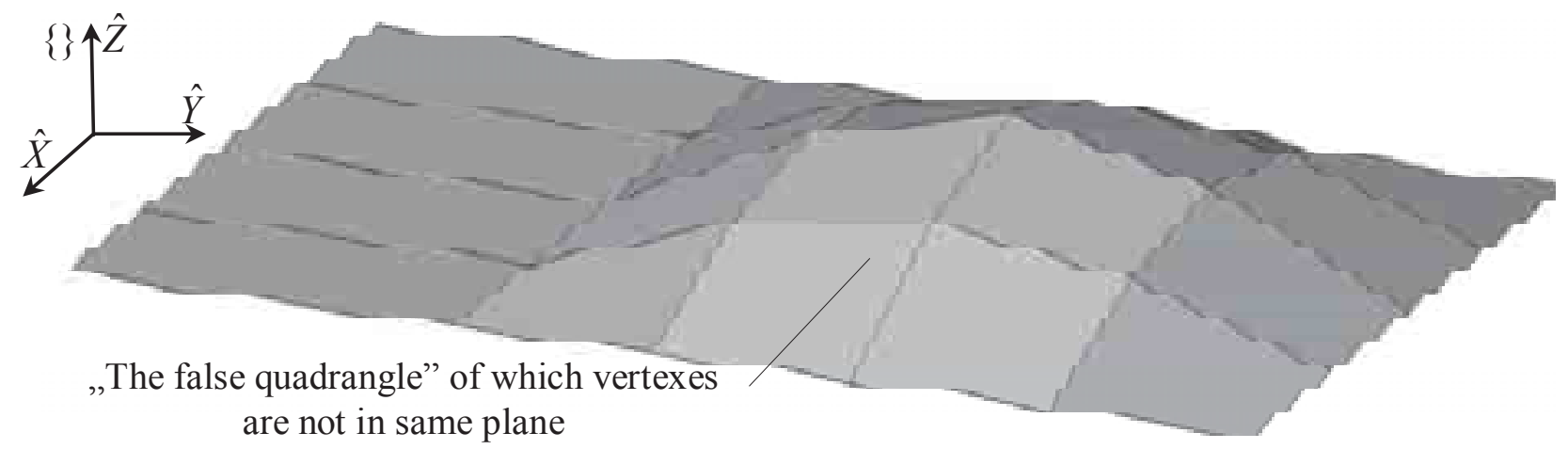

Fig. 8 Exemplary model of the surface area made of ,the false quadrangles"

If the surface area is made of quadrangles, then it is sufficient to choose any of their three vertexes to determine equation (2). Considering an additional possibility of building the discrete model, the road surface can be mapped in a more "flexible" way - using both quadrangles (e.g. rectangles) and triangles (Fig. 9).

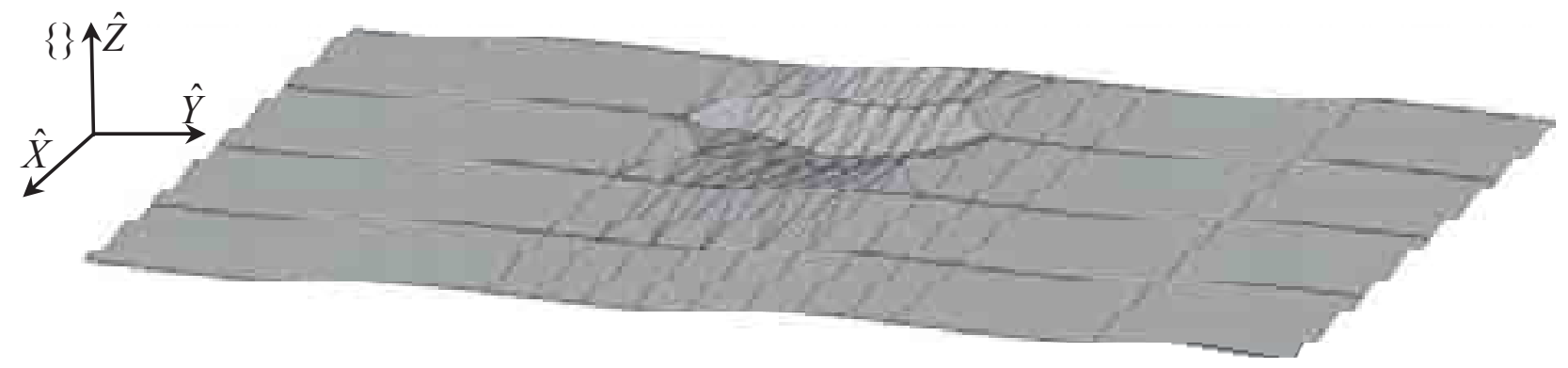

Fig. 9. Example of the road surface model made of the triangles and the rectangles

Then, some fragments of the road surface can be modelled by quadrangles, and if not, it can be done by triangles. In such a way, according to the authors, the road surface modelling can become more intuitive. 


\section{Computer simulations}

In order to represent particular models of the road surface, there were four computer simulations made. In each of them, the modelled vehicle moved over the surface of different shape. Models of the uneven road surface were made in the Blender environment, and then they were imported in the developed program. Each of the surface models were characterized by the parameters presented in Tab. 1.

Tab. 1. The road surface model - the assumed parameters

\begin{tabular}{|l|l|}
\hline Parameter & Description \\
\hline$x_{\min }, x_{\max }, y_{\min }, y_{\max }, z_{\min }, z_{\max }[\mathrm{m}]$ & $\begin{array}{l}\text { Maximum and minimum values of point coordinates of the road } \\
\text { surface model }\end{array}$ \\
\hline$u_{W P}$ & $\begin{array}{l}\text { A number of vertexes in the discrete model or a number of control } \\
\text { points in the continuous model }\end{array}$ \\
\hline$u_{T O}$ & $\begin{array}{l}\text { A number of triangles or rectangles in the discrete model or } \\
\text { a number of grid cells in the continuous model }\end{array}$ \\
\hline
\end{tabular}

The computer simulations were performed by the computer program, developed within the scope of the cited doctoral dissertation. The mathematical model of the vehicle used in the computer simulations is described in this work too.

\section{Example I}

The road surface model made is presented in Fig. 10. It consists of two flat fragments neighbouring to the projecting "bump". Since there are no surface slopes towards versor $\hat{Y}$ (of the system \{\} ), the whole surface model was made by the rectangles.

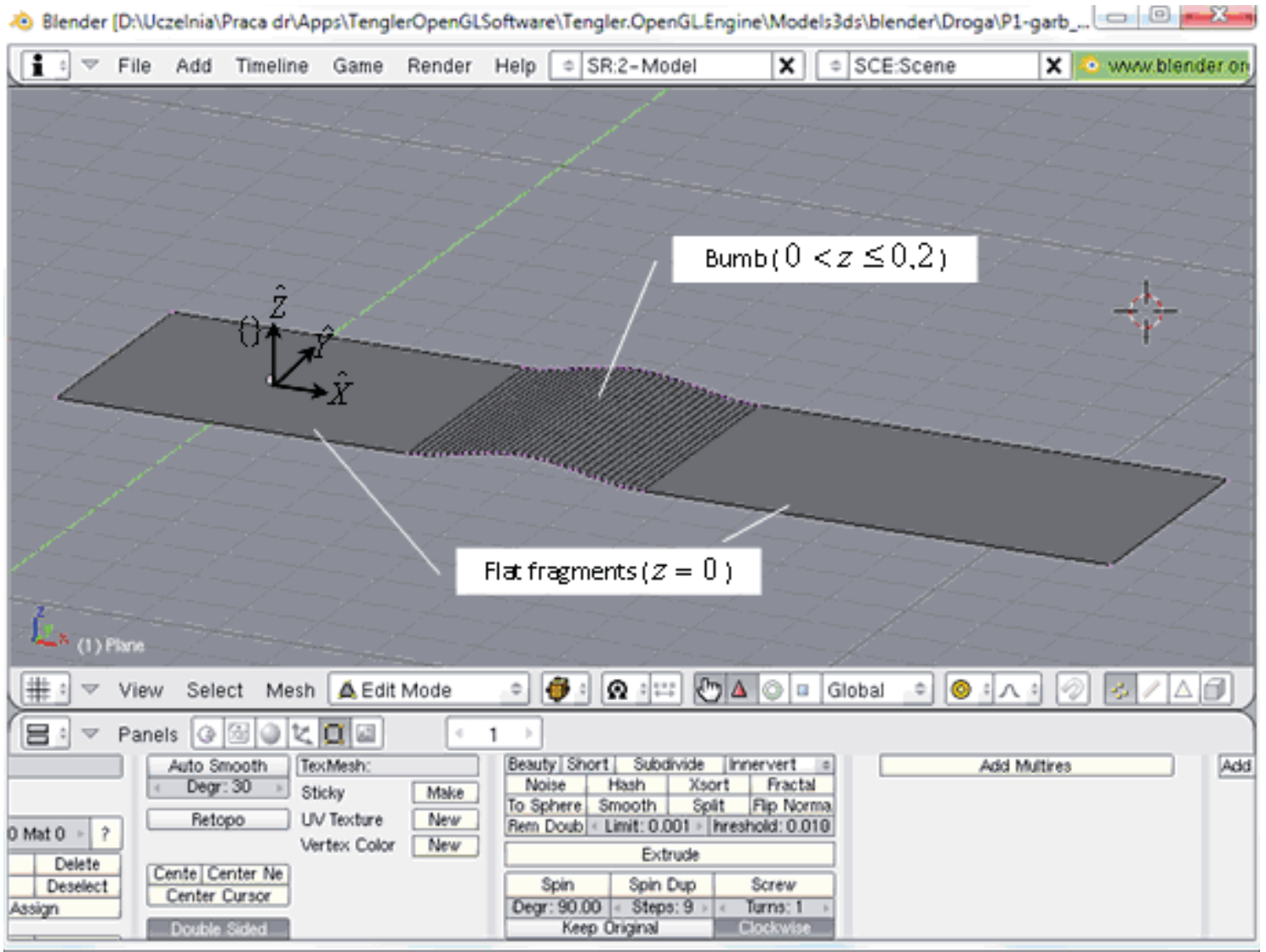

Fig. 10. Road surface model made in the Blender environment 
The values of the parameters, as in Tab. 1 are: $x_{\min }=-2, x_{\max }=11.5, y_{\min }=-2, y_{\max }=2$, $z_{\min }=0, z_{\max }=0.2, u_{W P}=40, u_{T O}=34$.

The surface model prepared in such a way was imported in the discrete form in the developed program.

A drive of the vehicle "straight on" over the projecting bump was simulated; and it is shown in the screen shots made during the animation (Fig. 11).
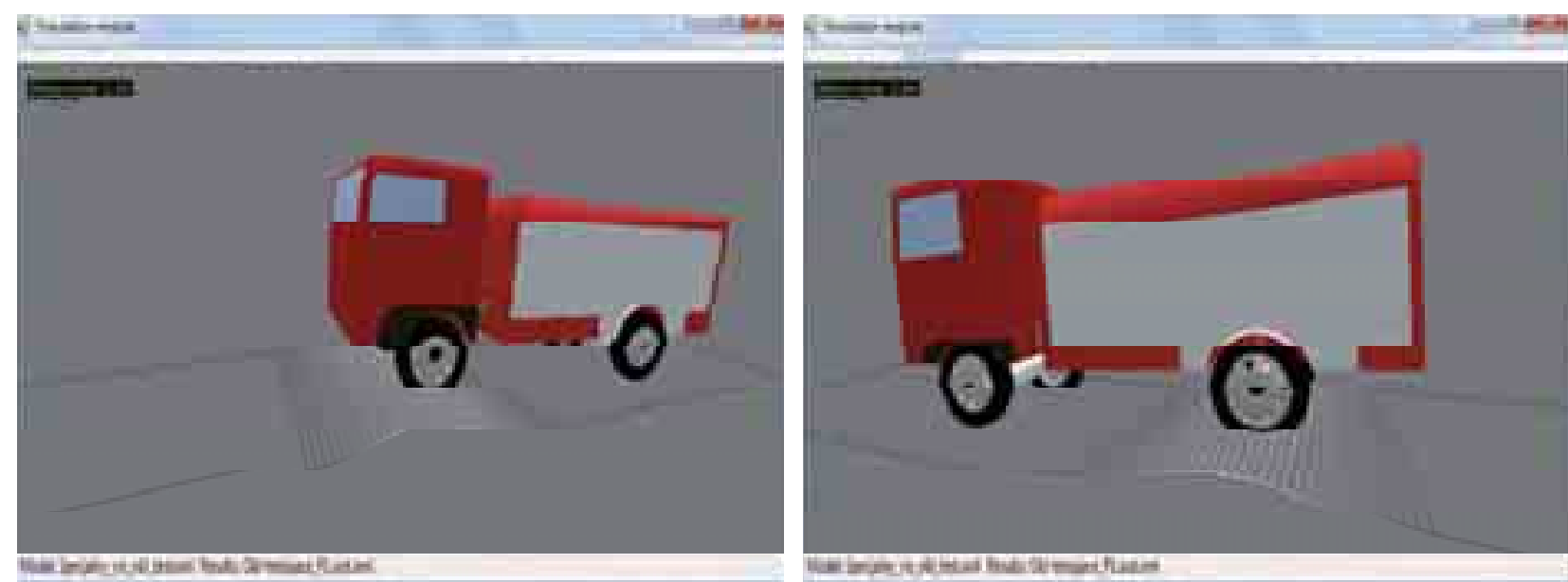

Fig. 11. Examples of the screen shots made during the animation

\section{Example II}

In this example, as in the previous one, the road surface model was prepared, and then it was imported as the discrete model in the developed program. The surface model made is characterized by a changeable profile both towards versor $\hat{X}$, and $\hat{Y}$ (Fig. 12).

The parameter values of the presented model, as in Tab. 1, are: $x_{\min }=-2.5, x_{\max }=12.7$, $y_{\min }=-5.5, y_{\max }=3.5, z_{\min }=0, z_{\max }=0.47, u_{W P}=207, u_{T O}=344-$ unlike in the model used in the first example, the number of quadrangles is greater than the number of the vertexes.

Examples of the screen shots made during the animation, presenting the track over which the vehicle moved, are presented in Fig. 13.

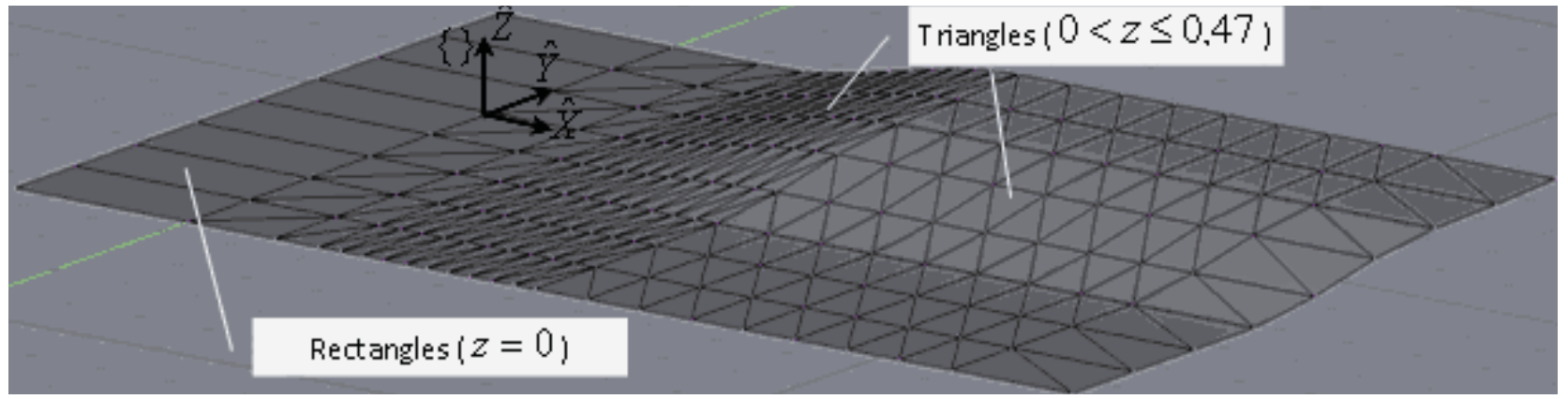

Fig. 12. Road surface model

Example III

Within the scope of another simulation, the model of the road surface, presented in Fig. 14, was made. This model was imported as the discrete model in the developed program.

Values of the appropriate parameters of the assumed road surface model are: $x_{\min }=-2, x_{\max }=51$, $y_{\min }=-9, y_{\max }=9, z_{\min }=-2, z_{\max }=0, u_{W P}=633, u_{T O}=1104$.

The screen shots presenting the track of the moving vehicle are presented in Fig. 15. 

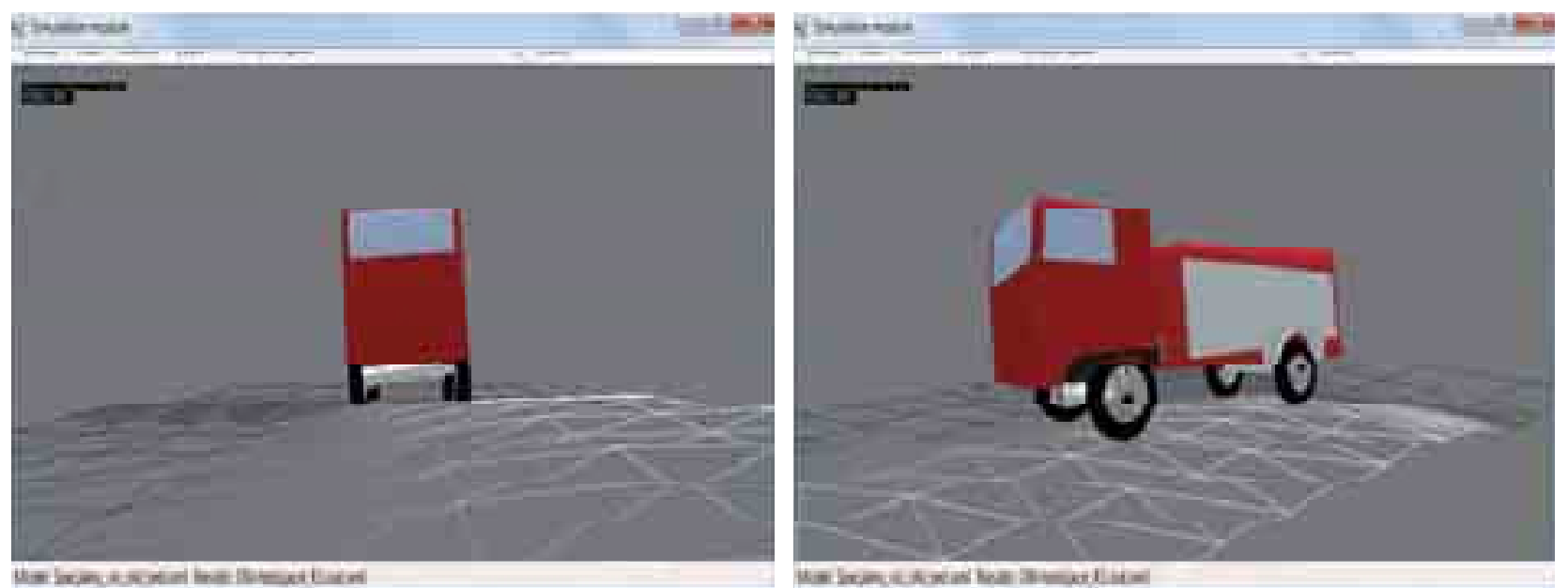

Fig. 13. Examples of the screen shots made during the animation

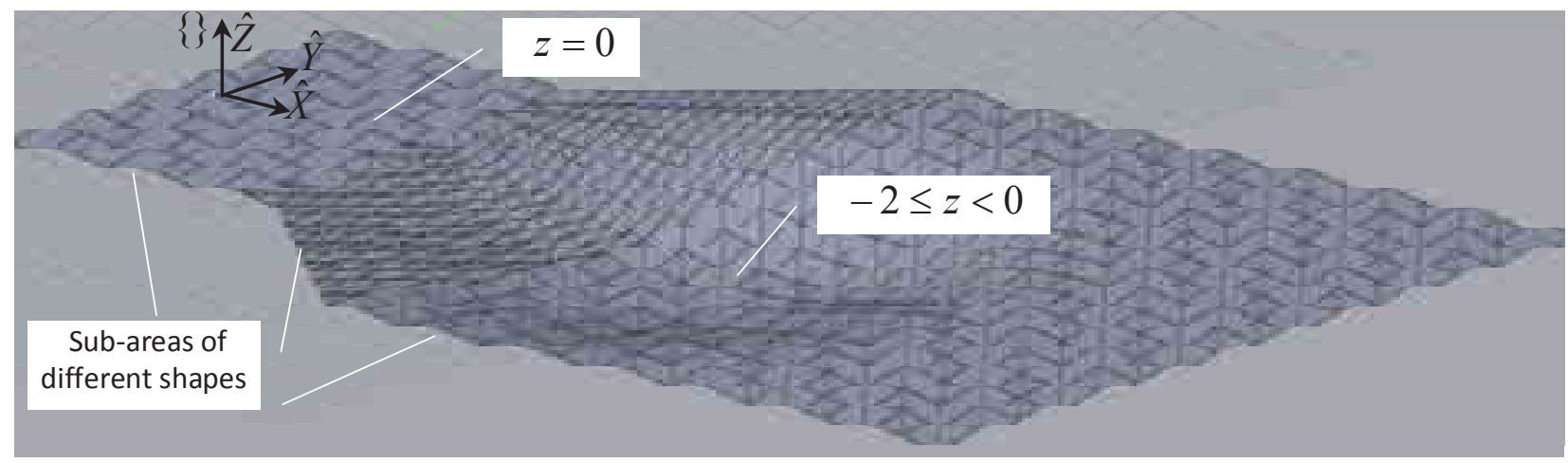

Fig. 14. Road surface model
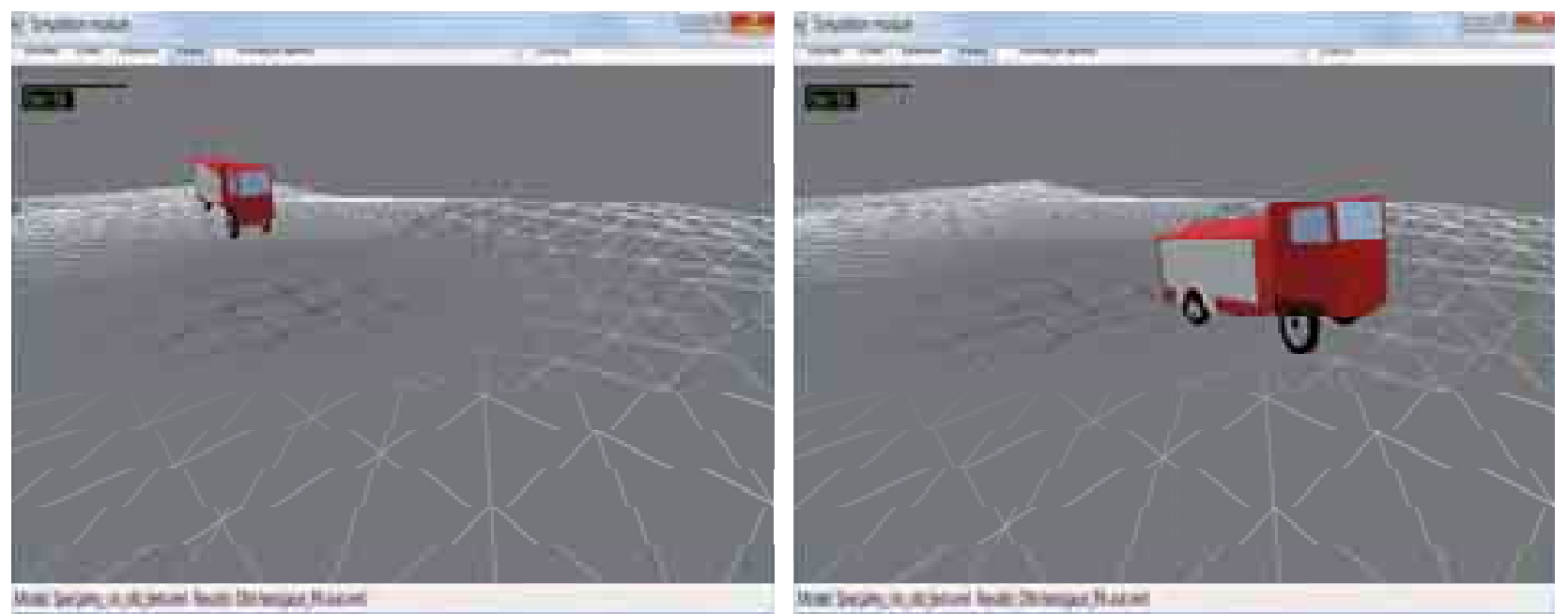

Fig. 15. Examples of the screen shots made during the animation

\section{Example IV}

In the last example, the road surface model was prepared, and the model was imported as the continuous model in the developed program. The grid of the control points of this model is presented in Fig. 16.

Values of the parameters of the presented surface model are following: $x_{\min }=-2, x_{\max }=49$, $y_{\min }=-8, y_{\max }=8, z_{\min }=-0.8, z_{\max }=1.2, u_{W P}=128, u_{T O}=165$.

Selected phases of the modelled vehicle drive are presented in Fig. 17. 


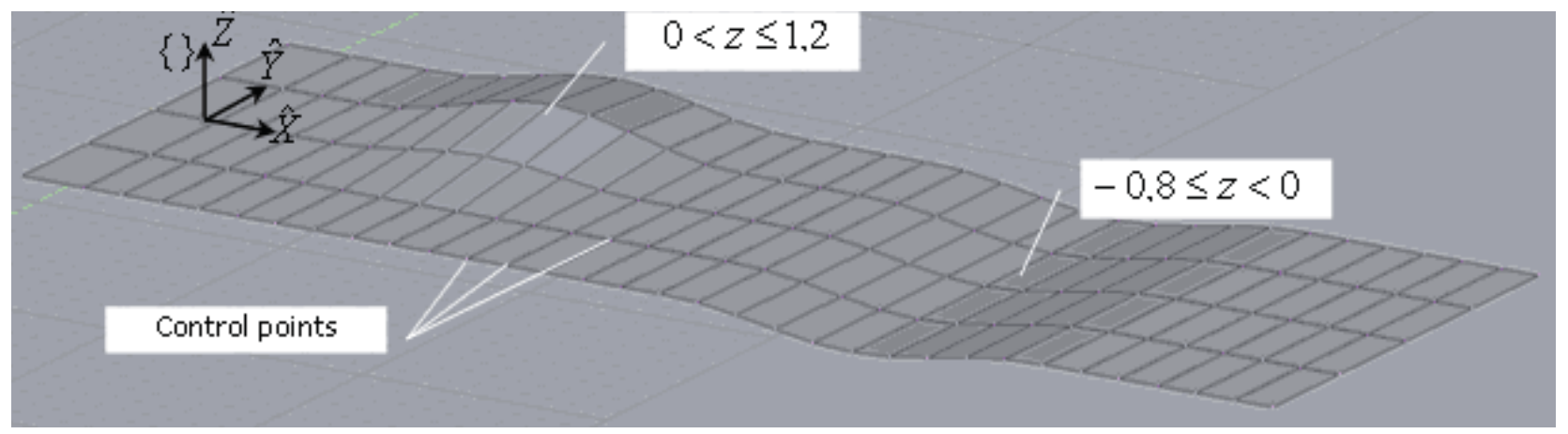

Fig. 16 Control point grid of the continuous model of the road surface
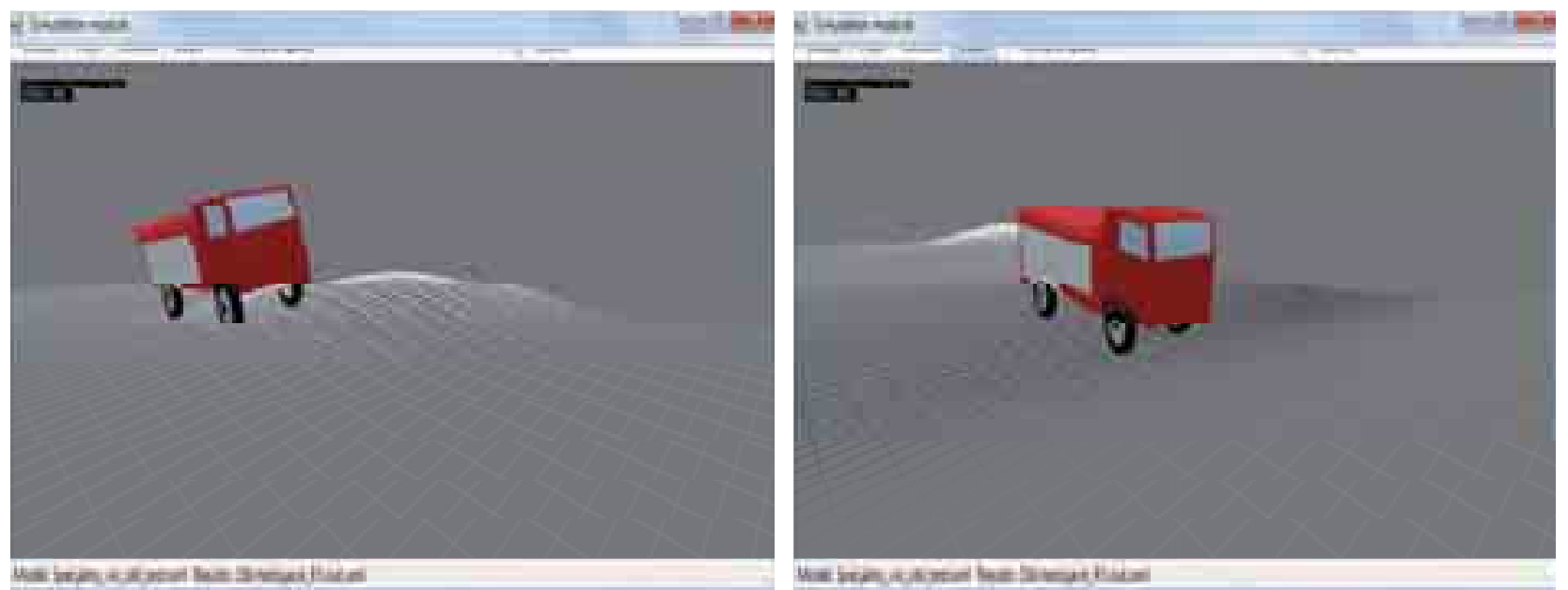

Fig. 17. Examples of the shot screens made during the animation.

\section{Conclusions}

The computer simulations presented in this article, are only a part of works performed by the authors. The presented models of the road surface enable to conduct a series of other computer simulations, which can include different types of the road surface unevenness. For example, in doctoral dissertation [5], a simulation of a drive of the vehicle over smooth and sharp unevenness was described, and the calculation results were compared with the results obtained in an experimental way - for this purpose there were experiments carried out using specialist measurement equipment. Additionally, in work [6] the authors determined the essential parameters of the vehicle, including an inclination angle of the road surface, at which a loss of its stability takes place.

The main advantage of the discrete model of the road surface is a possibility to take its unevenness into account together with the neighbouring flat areas - this case was presented in example I. It is difficult to achieve in the continuous model - because the grid of the control points has to be compacted appropriately in the points where there are flat fragments. An additional advantage of the discrete model is a possibility to obtain fragments of the surface of different shapes (Fig. 14) in the continuous model, due to the point grid structure, it is impossible. While analyzing advantages of the continuous model, it was found that courses of the results of the calculations, made by its use, had a "smoother" character (no "sharp oscillations") than in the case of the discrete model. This effect can be obtained in the discrete model by compacting the division into polygons, although it extends the calculation time.

In this article, the authors conducted also an analysis of the time of calculations made using the discrete model, compacting the division into polygons. For example, by increasing a number of the polygons four times in the first example presented, the obtained calculations times were only 2.5 
longer than in the case of the non-compacted road surface model. Such a relationship of the calculation time with reference to the density of the division of the modelled road surface into the polygons confirms applicability of the NNS algorithm.

\section{Acknowledgements}

The investigation was supported by the National Science Centre in Cracow under doctoral research grant 0630/B/T02/2011/40.

\section{References}

[1] Dziubiński, I., Siewierski, L., Mathematics for technical universities (in Polish), PWN, Warszawa 1989.

[2] Friedman, J. H., Bentley, J. L., Finkel, R. A., An Algorithm for Finding Best Matches in Logarithmic Expected Time, ACM Transactions on Mathematical Software (TOMS), Vol. 3, pp. 209-226, New York 1977.

[3] Keys, R., Cubic convolution interpolation for digital image processing, Acoustics, Speech and Signal Processing, Vol. 29, pp. 1153-1160, 1981.

[4] Samet, H., The design and analysis of spatial data structures, Addison-Wesley Longman Publishing Co., Boston 1990.

[5] Tengler, S., Analysis of dynamics of special vehicles with high gravity centre, $\mathrm{PhD}$ Thesis, Faculty of Mechanical Engineering and Computer Science, University of Bielsko-Biala, 2012.

[6] Tengler, S., Harlecki, A., Analysis of dynamics of special vehicles with high gravity centrethe case of stability loss, Int. J. of Applied Mechanics and Engineering, in print. 